\title{
Comparison of Efficacy Between Triamcinolone Acetonide and Triamcinolone Hexacetonide for Intraarticular Therapy in Juvenile Idiopathic Arthritis: A Retrospective Analysis
}

Deirdre De Ranieri ( $\square$ DDeRanieri@luriechildrens.org )

Lurie Children's Hospital

Angela Chun

Baylor College of Medicine

Lutfiyya Muhammad

Northwestern University

\section{Research Article}

Keywords: Injections, Intraarticular, Corticosteroids, Juvenile Idiopathic Arthritis, Triamcinolone Acetonide, Triamcinolone Hexacetonide

Posted Date: September 27th, 2021

DOl: https://doi.org/10.21203/rs.3.rs-894105/v1

License: (c) (1) This work is licensed under a Creative Commons Attribution 4.0 International License.

Read Full License 


\section{Abstract \\ Background}

There are many FDA-approved corticosteroid preparations available for intra-articular injection, however triamcinolone hexacetonide is not one of them. It was the intraarticular drug of choice among pediatric rheumatologists up until approximately a decade ago, when production of this medication ceased. It can be obtained in the United States and Canada via importation from Europe, but it is not FDA-approved at this time. We wish to compare the duration of remission of intraarticular triamcinolone hexacetonide (TH) with that of triamcinolone acetonide (TA) in children with Juvenile Idiopathic Arthritis (JIA) and demonstrate its safety in this population.

\section{Methods}

This retrospective chart review included 39 patients with JIA who received intraarticular corticosteroid injections (IACls) from September 2018 to September 2019. These patients were reviewed and their lifetime injections with either TH (41 joints) or TA (124 joints) was noted through May 30, 2021. Patients with concomitant systemic therapy initiation were excluded. The primary outcome was time to relapse. Relapse was defined by the presence of arthritis on physical examination by an attending rheumatologist. Kaplan-Meier curves and a log-rank test were constructed to compare the probability of time to relapse between IACI injections. Additionally, mixed effects cox regression models were constructed to account for multiple injections per participant.

\section{Results}

Kaplan-Meier estimator of median relapse time in months was higher for $\mathrm{TH}$. Based on the log-rank test, TA joints had a higher probability of experiencing a relapse during the study time ( $p$-value $<0.001)$. The hazard of time to relapse was reduced when comparing TH to TA in both unadjusted and adjusted mixed effects cox regression models (unadjusted hazard ratio (95\% confidence interval): $0.184(0.089,0.381)$; adjusted hazard ratio (95\% confidence interval): $0.189(0.092,0.386))$.

\section{Conclusions}

TH has longer duration of action than TA and is associated with less systemic side effects. It should be considered the drug of choice for intraarticular corticosteroid injections in children with JIA.

\section{Background}

Intraarticular corticosteroid injections (IACls) have long been considered a safe and effective treatment option in the management of patients with Juvenile Idiopathic Arthritis $(\mathrm{JIA})^{1-4}$. IACls are used to achieve 
rapid resolution of arthritis, with the goals of providing pain relief and preventing joint damage $\mathrm{e}^{1}$. The use of IACls is associated with significantly less systemic toxicity than taking corticosteroids orally, intramuscularly, or intravenously ${ }^{5,6}$. Intraarticular corticosteroids are often used as initial therapy in patients with Oligoarticular JIA and as adjunctive therapy in patients with other subtypes of JIA who may be flaring in a limited number of joints or either initiating or transitioning between different systemic therapies ${ }^{1,7}$. In JIA, several studies have reported long term benefits of IACls, including long term reduction in pain and inflammation ${ }^{1,8}$, stabilization and/or prevention of limb length discrepancy ${ }^{3,4}$, as well as resolution of joint pannus ${ }^{9}$.

There are several different intraarticular corticosteroid formulations available. Lower solubility agents have been shown to have slower absorption and longer duration of action, leading to higher efficacy ${ }^{1,10}$. Triamcinolone acetonide (TA) and triamcinolone hexacetonide $(T H)$ are the most commonly used formulations of injectable corticosteroids in North America and Europe ${ }^{11}$. TA and TH differ only in the presence of one side chain, but their efficacy is markedly different. Several studies have shown TH to be superior to TA in duration of clinical remission in patients with $\mathrm{JIA}^{1,10-13}$. This could potentially be due to its slower absorption and release ${ }^{10}$. Additionally, $\mathrm{TH}$ has been shown to specifically reduce synovial $\mathrm{T}$ cell lymphocytes with associated decrease in inflammatory cytokines ${ }^{14,15}$.

The commercial production of TH (labelled as Aristospan) in the United States was halted in 2015. Due to the lack of availability of Aristospan, pediatric rheumatologists returned to using other steroid formulations, such as TA, for intraarticular injections. Another brand of TH (labelled as Lederspan) became available to patients who have failed TA injections through the Personal Importation Policy (PIP) as set forth by the Food and Drug Administration (FDA). The PIP allows the importation of certain medications for which there are no currently FDA-approved acceptable alternatives in the United States and the patient has failed the similar FDA-approved available medication. At Ann \& Robert H Lurie Children's Hospital, we were granted access to this medication and have been using it for over two years in patients who have failed TA, as evidenced by a flare in arthritis.

In order to compare the efficacy of TH to the current standard of TA in maintaining clinical remission for participants with JIA, we performed a retrospective study to compare time to relapse between TH and TA. Our primary outcome was time to relapse based on patient report and physician's clinical exam.

Additionally, since the knees were the most commonly injected joints in children with JIA, we performed a subgroup analysis to assess time to relapse for participants that received IACls into the knee joint only.

\section{Methods}

This protocol was reviewed and approved by the Institutional Review Board at Ann \& Robert H Lurie Children's Hospital (IRB 2019-2951). In this retrospective chart review, EMR CPT codes identified patients, diagnosed with JIA according to the International League of Associations for Rheumatology (ILAR) criteria $^{16}$, who received an IACI with either TA or TH at a single tertiary center between September 1, 2018 
and September 1, 2019. These patients were reviewed, and their life-time injections through May 30, 2021, were included in the analysis. Patients with systemic JIA and undifferentiated arthritis were excluded. To minimize confounding bias, patients who had received an IACl within 3 months prior to the injection or those who had started a new systemic medication or were transitioning between systemic medications within 3 months of the injection were excluded. Medical records were reviewed for patient demographics and clinical course.

Lederspan (a brand of TH) is a German medication that is not currently FDA approved, so it was obtained via the Personal Importation Policy (PIP), which allows a patient to import a foreign non-FDA approved medication if they have failed the available FDA-approved medication (TA). All of the patients in this study had failed TA and were thus eligible for TH based on this policy. The medication was obtained from Germany via the Canadian pharmaceutical company, Medexus Pharma.

Ultrasound guided IACls were performed by the same provider using standard techniques, either with topical anesthesia (using a J-tip, which is a sterile, single use, subcutaneous needle-less injection device) or under sedation with an anesthesiologist. Standardization of dosages for TA were as follows: large joints (knees, hips, shoulders) received 60-80mg depending on the size of the patient; medium joints (ankles, wrists, elbow) received 40mg; and small joints (fingers, toes) received 4-8mg. Standardized dosages for TH were as follows: large joints (knees, hips, shoulders) received 40mg; medium joints (ankles, elbow) received 30mg, the wrist received 20mg; and small joints (fingers, toes) received 4-6mg. After IACl, patients who had knees and hips injected were advised to minimize their activity for a period of at least 24 hours, and up to 48 hours, as this has been shown in adults to improve outcome in larger joints ${ }^{2,17-19}$.

Relapse was defined by the presence of active arthritis in the joint per an attending pediatric rheumatologist's physical exam. Active arthritis was defined as swelling within the joint, and if no appreciable swelling was present, other signs and symptoms suggestive of arthritis such as limitation in range of motion, pain with movement of the joint, and inflammatory type symptoms such as morning stiffness, were used to assess activity.

Demographics and patient characteristics were compared between IACl types using the Mann-Whitney U test for non-normal continuous variables and Fisher's exact test for categorical variables. Time to relapse in months was the primary outcome in our analyses. Kaplan-Meier curves and log-rank tests with all joints and knees only were constructed to compare the probability of time to relapse between IACl groups. Participants were censored if they did not experience a relapse during the study duration. Unadjusted and adjusted mixed effects cox regression models were constructed to account for multiple injections per participant. IACl type (TA vs. TH) was the primary predictor in the models. The adjusted model for all joint locations included age, sex, ethnicity, JIA diagnosis categories, and joint injection location. The covariates in the adjusted model for knee-only joint injections were age, sex, ethnicity, and JIA diagnosis categories. Hazard ratios (HRs) and $95 \%$ confidence intervals (Cl) of the HRs summarized the findings from the mixed effects cox regression models. 


\section{Results}

Demographics and participant characteristics are listed in Table 1. 
Table 1

Demographics and Participant Characteristics by IAC injection type. Total sample size is 39 participants with 165 injections. Nine of the 39 participants received both IAC injection types. P-values from Mann-Whitney U tests and Fisher's Exact tests are listed are for the comparison between IAC injection types. *Denotes $\mathrm{p}$-value $<0.05$. Abbreviation: IQR $=$ Interquartile range

\begin{tabular}{|c|c|c|c|}
\hline & TA & TH & \\
\hline & $N=34$ with & $N=14$ with & $\mathrm{p}$-value \\
\hline & 124 injections & 41 injections & \\
\hline Relapse time in months (median [IQR]) & $3.00[2.00,6.00]$ & $11.00[5.00,18.00]$ & $<0.001^{*}$ \\
\hline Age in years (median [IQR]) & $4.88[2.92,10.65]$ & $4.83[3.02,7.67]$ & 0.901 \\
\hline JIA Disease Duration in years (median [IQR]) & $0.46[0.19,2.66]$ & $1.17[0.44,4.33]$ & 0.179 \\
\hline Female (\%) & $27(79.4)$ & $10(71.4)$ & 0.708 \\
\hline Ethnicity (\%) & & & 0.160 \\
\hline White & $27(79.4)$ & $13(92.9)$ & \\
\hline Black & $4(11.8)$ & $0(0.0)$ & \\
\hline Hispanic & $3(8.8)$ & $0(0.0)$ & \\
\hline Unknown & $0(0.0)$ & $1(7.1)$ & \\
\hline JIA Diagnosis (\%) & & & 0.461 \\
\hline Oligo & $24(70.6)$ & $12(85.7)$ & \\
\hline Poly & $8(23.5)$ & $1(7.1)$ & \\
\hline Psoriatic & $2(5.9)$ & $1(7.1)$ & \\
\hline Injections by Joint Locations (\%) & & & 0.051 \\
\hline Knee & $62(50.0)$ & $24(58.5)$ & \\
\hline Ankle & $31(25.0)$ & $5(12.2)$ & \\
\hline Wrist & $14(11.3)$ & $1(2.4)$ & \\
\hline Elbow & $2(1.6)$ & $1(2.4)$ & \\
\hline Fingers/Toes & $14(11.3)$ & $9(22.0)$ & \\
\hline Hip & $1(0.8)$ & $0(0.0)$ & \\
\hline Mid-Foot & $0(0.0)$ & $1(2.4)$ & \\
\hline
\end{tabular}


A total of $39 \mathrm{JIA}$ patients with 165 IACls are included in this study. Nine of the 39 participants received both injections. There were 34 participants that received 124 TA injections, and 14 participants received $41 \mathrm{TH}$ injections. Median relapse time in months for the TH group was significantly longer than the TA group (median TA relapse time $=3$ and median TH relapse time $=11$, Mann-Whitney $U$ test $p$-value $<$ 0.001). The median age in both groups were approximately the same (TA median age $=4.88$ and $\mathrm{TH}$ median age $=4.83$, Mann-Whitney $U$ test $p$-value $=0.901)$. Median JIA disease duration in years at time of injection was not statistically different between groups (TA median JIA disease duration $=0.46$ and $\mathrm{TH}$ median JIA disease duration = 1.17, Mann-Whitney U p-value $=0.179$ ). In both groups, there were more females than males. There were no statistical differences between groups for ethnicity, JIA diagnosis subtype, and injections by joint locations. For both $\mathrm{TA}$ and $\mathrm{TH}$, the knee was the most frequently injected joint.

Figures 1 and 2 illustrates the Kaplan-Meier plots for all injections and knee only injections respectively.

The Kaplan-Meier estimator of the median relapse time in months was higher for TH in comparison to TA (TH median = 18 and TA median = 4). Based on the log-rank test, there was a difference between TA and $\mathrm{TH}$ in the probability of experiencing a relapse during the study time ( $\mathrm{p}$-value $<0.001)$. For the knee only analysis, the Kaplan-Meier estimator of the median relapse time for the TH group was 14 months while the Kaplan-Meier estimator of the median relapse time was 3 months for those that received TA. There was a difference between TA and TH knee injections in the probability of experiencing a relapse during the study period (log-rank p-value $<0.001$ ).

The adjusted mixed effects cox regression model results for all injections and knee only injections are in Tables 2 and 3 respectively. 
Table 2

Hazard ratios from mixed effects cox regression models that included injections in all joints. Sample size is 39 participants with 165 injections. Nine participants received both IAC injection types. The mixed effects cox model included age, sex, ethnicity, diagnosis (oligo vs. other (poly, psoriatic, and ERA)), and joints (knee vs. other

(ankle, wrist, elbow, finger/toes, shoulder, hip, SI, and TMJ)) as covariates. *Denotes $p$-value $<0.05$.

\begin{tabular}{|lll|}
\hline & $\begin{array}{l}\text { Hazards Ratio } \\
(\mathbf{9 5 \%} \mathbf{~ C l})\end{array}$ & p-value \\
\hline Injection: TH vs TA & $0.189(0.092,0.386)$ & $<0.001^{\star}$ \\
\hline Age in years & $0.996(0.893,1.112)$ & 0.950 \\
\hline Females vs Males & $0.555(0.177,1.743)$ & 0.310 \\
\hline JIA Diagnosis: Oligo vs Other & $0.346(0.127,0.943)$ & $0.038^{*}$ \\
\hline Joint: Knee vs Other & $1.413(0.918,2.174)$ & 0.120 \\
\hline Ethnicity: White vs Other & $0.439(0.116,1.659)$ & 0.220 \\
\hline
\end{tabular}

Table 3

Hazard ratios from mixed effects cox regression models with participants that received IAC injections in the knee only. Sample size is 21 participants with 86 injections, and nine participants received both IAC injection types. The mixed effects cox model included age, sex, ethnicity, and JIA diagnosis (oligo vs. other (poly, psoriatic, and ERA)) as covariates. *Denotes $p$-value $<0.05$.

\begin{tabular}{|lll|}
\hline & $\begin{array}{l}\text { Hazards Ratio } \\
(\mathbf{9 5 \%} \mathbf{~ C l})\end{array}$ & p-value \\
\hline Injection: TH vs TA & $0.131(0.052,0.322)$ & $<0.001^{*}$ \\
\hline Age in years & $0.946(0.831,1.077)$ & 0.400 \\
\hline Females vs Males & $0.407(0.104,1.593)$ & 0.200 \\
\hline JIA Diagnosis: Oligo vs Other & $0.208(0.055,0.782)$ & $0.020^{*}$ \\
\hline Ethnicity: White vs Other & $0.536(0.010,2.882)$ & 0.470 \\
\hline
\end{tabular}

The hazard of time to relapse was reduced when comparing the TH group to TA group in both the unadjusted and adjusted mixed effects cox regression models (unadjusted $\mathrm{HR}=0.184,95 \% \mathrm{Cl}(0.089$, 0.381), $\mathrm{p}$-value $<0.001$; adjusted $\mathrm{HR}=0.189,95 \% \mathrm{Cl}(0.092,0.386), \mathrm{p}$-value $<0.001)$. The reduced hazard implies that time to relapse was longer for the TH group relative to the TA group. Similarly, participants in 
the knee injections only analysis that received TH had a longer relapse time than those that received TA. The estimated unadjusted and adjusted HRs that compared TH to TA for those that received knee injections only were both statistically significant (unadjusted $\mathrm{HR}=0.109,95 \% \mathrm{Cl}(0.043,0.278)$, $\mathrm{p}$-value < 0.001 ; adjusted $\mathrm{HR}=0.131,95 \% \mathrm{Cl}(0.052,0.322), \mathrm{p}$-value $<0.001)$.

\section{Discussion}

Intraarticular corticosteroid injections (IACls) are often used as first-line therapy in patients with Oligoarticular JIA, in whom a limited number of joints are affected. IACls are also used as adjunctive therapy in other subtypes of JIA to expeditiously control inflammation and decrease pain while awaiting systemic medications to take effect. IACls have the benefit of quickly decreasing inflammation, thus minimizing the risk of morbidity associated with JIA, such as cartilage destruction, muscle wasting, and

leg length discrepancies ${ }^{1-4,14}$. Acquired leg length discrepancies in children with JIA are thought to result from stimulation of the growth plate due to the inherent hyperemia in the region secondary to synovial inflammation ${ }^{4}$. Intraarticular corticosteroids have been shown to effectively reduce synovial T lymphocytes and downregulate certain pro-inflammatory cytokines, including TNF-a, IL-1b, extranuclear HMGB-1, ICAM-1, and VEGF ${ }^{15}$.

Historically, TH has been shown in several studies to have a longer duration of action and a superior side effect profile compared to other intraarticular corticosteroid formulations ${ }^{10-13,20}$. TH was superior, even at lower doses, with effects lasting up to 24 months ${ }^{10,11}$. Furthermore, it has been shown to be an effective therapy for inflammatory arthritis in all subtypes of $\mathrm{JIA}^{21}$. Aristospan, a formulation of triamcinolone hexacetonide $(\mathrm{TH})$, was the preferred corticosteroid for intraarticular injection until it became unavailable in the United States almost a decade ago. Since that time, pediatric rheumatologists have been using Kenalog, a brand of triamcinolone acetonide (TA). However, there have been few contemporary studies comparing the efficacy of the two.

The results from our study support the past comparative studies in pediatric rheumatology that have suggested superiority of TH compared to TA. The median time to flare in joints treated with TH was 11 months in our study, which is similar to the mean time to flare reported by Eberhard et al. of $10.14 \pm 0.49$ months in the group injected with $\mathrm{TH}^{13}$. Lepore et al. reported a mean duration of remission of 13.9 months in knees injected with TH for patients with oligoarticular $\mathrm{JIA}^{22}$. This slightly longer duration of remission may be secondary to the isolation of knee injections, with previous studies suggesting IACls were most effective in this joint ${ }^{8,13,21}$. Similar to us, both Eberhard et al. and Lepore et al. defined remission as a complete disappearance of clinical signs of inflammation. Other studies have reported much longer durations of remission. Zulian et al. compared the efficacy of TH and TA in oligoarticular JIA in a prospective study, with almost double the response rate with $\mathrm{TH}$ at 24 months ${ }^{10}$. Subsequently, Zulian et al. compared TA at twice the dose of TH in children with symmetric arthritis in a prospective double-blinded study, with similar findings ${ }^{11}$. However, the scale to assess for arthritis was different, allowing for a nominal degree of arthritis. In a retrospective study, Marti et al. reported a longer median 
duration of remission of 23.1 months for patients who underwent IACl with either TH or TA, but patients were often started on concomitant medications at time of injection ${ }^{8}$.

The median time to flare in joints treated with TA in our study was 3 months, which is shorter than the mean time to flare of $7.75 \pm 0.49$ months reported by Eberhard et al. However, it should be noted that smaller doses were often used in our patients compared to the doses used by Eberhard et al., who used $80 \mathrm{mg}$ for the knee and $60 \mathrm{mg}$ for the elbow, ankle, and wrist, and some studies suggest that higher doses of TA are needed to be effective $\mathrm{i}^{10,11,13}$. Additionally, all of the patients in our study had previously failed $\mathrm{IACl}$ with $\mathrm{TA}$, suggesting a more refractory disease group and the potential for a more robust response if initially treated with $\mathrm{TH}$.

Side effects of $\mathrm{IACl}$ are usually mild and temporary, with discomfort at the injection site being the most common ${ }^{23}$. Other reported side effects include mood and sleep alteration, appetite changes, menstrual irregularities, weight gain, and Cushingoid appearance, especially in young patients or in those getting multiple injections ${ }^{24,25}$. Skin hypopigmentation and subcutaneous atrophy from leakage of the steroid along the needle track can also be seen, but these changes usually resolve with time ${ }^{1,25}$. In our cohort, the use of $\mathrm{TH}$ for IACl was associated with more post-procedural discomfort, but fewer systemic side effects, likely due to increased intraarticular residence and less systemic absorption ${ }^{25}$. Compared to those who received TA, those who received TH had less post-procedural emotional lability, appetite changes and weight gain, flushing and malaise.

Limitations of this study include the small sample size and the retrospective nature of chart reviews. The sample size was limited by the availability of the medication, as the cost was not covered by insurance. Furthermore, there was often parental hesitance to use a medication that was not FDA-approved. Interestingly, more males than females received TH compared to TA, and the reason for this is unclear, i.e., if the medication was offered to more male patients, or if the parents of male patients were more likely to want to try it (as opposed to starting systemic therapy). We know that in this age group, in general, there is a female predominance, so that will be an interesting variable to evaluate going forward.

Additionally, by excluding patients who concomitantly started or changed systemic therapy, we may have introduced some selection bias, as there was no standardization for stepwise escalation of therapy. This could suggest that patients with worse disease may have been quickly started on concomitant therapies and excluded from the study. However, we would argue that since all patients were required to have previously failed TA injections to qualify for TH injections, this may suggest that they had more severe or refractory disease at baseline.

A future goal is to conduct a prospective study evaluating the duration of remission of TH versus TA, when TH is used in steroid-naïve joints as opposed to being used as a second-line agent for intraarticular corticosteroid injections. 
In conclusion, we confirm the finding that TH is superior to TA in terms of time to flare and associated side effects, and we advocate for increased availability of this product for our patients. At the time of this writing, Medexus Pharma is the sole pharmaceutical company with rights to sell this drug in the United States. They are currently importing Trispan from France, which is the same chemical formulation as Lederspan which was imported from Germany. Literature to support the superiority of TH over TA could expedite the FDA approval of this medication and render it less cumbersome for pediatric rheumatologists to obtain.

\section{List Of Abreviations}

JIA: Juvenile Idiopathic Arthritis

TA: Triamcinolone Acetonide

TH: Triamcinolone Hexacetonide

FDA: Food \& Drug Administration

IACl: Intraarticular Corticosteroid Injection

ILAR: International League Against Rheumatism

EMR: Electronic Medical Record

CPT: Current Procedural Terminology

\section{Declarations}

Ethics approval and consent to participate: The study was conducted according to the guidelines of the Declaration of Helsinki, and approved by the Institutional Review Board at Ann \& H. Lurie Children's Hospital of Chicago (IRB 2019-2951)

Consent for publication: Not applicable

Availability of data and materials: Data sharing is not applicable to this article as no datasets were generated or analyzed during the current study.

Competing interests: The authors declare that they have no competing interests

Funding: The source(s) of support in the form of grants or industrial support: Research reported in this publication was supported by Fostering Innovative Rheumatic Disease Team-Based Research to Improve Daily Life (FIRST-DailyLife), an NIH/NIAMS funded center (P30AR072579). 
Authors' contributions: DD, AC, and LM conceived and designed the study; LM performed the statistical analysis; DD and AC collected data; DD, AC and LM drafted the manuscript and revised the manuscript.

Acknowledgements: Not applicable

\section{References}

1. Cleary AG, Murphy HD, Davidson JE. Intraarticular corticosteroid injections in juvenile idiopathic arthritis. Arch Dis Child 2003;88:192-6.

2. Dent $\mathrm{PB}$, Walker N. Intraarticular corticosteroids in the treatment of juvenile rheumatoid arthritis. Curr Opin Rheumatol 1998;10:475-80.

3. Padeh S, Passwell JH. Intraarticular corticosteroid injection in the management of children with chronic arthritis. Arthritis Rheum 1998;41:1210-4.

4. Sherry DD, Stein LD, Reed AM, Schanberg LE, Kredich DW. Prevention of leg length discrepancy in young children with pauciarticular juvenile rheumatoid arthritis by treatment with intraarticular steroids. Arthritis Rheum 1999;42:2330-4.

5. Allen RC, Gross KR, Laxer RM, Malleson PN, Beauchamp RD, Petty RE. Intraarticular triamcinolone hexacetonide in the management of chronic arthritis in children. Arthritis Rheum 1986;29:997-1001.

6. Furtado RN, Oliveira LM, Natour J. Polyarticular corticosteroid injection versus systemic administration in treatment of rheumatoid arthritis patients: a randomized controlled study. $\mathrm{J}$ Rheumatol 2005;32:1691-8.

7. Bloom BJ, Alario AJ, Miller LC. Intraarticular corticosteroid therapy for juvenile idiopathic arthritis: report of an experiential cohort and literature review. Rheumatol Int 2011;31:749-56.

8. Marti P, Molinari L, Bolt IB, Seger R, Saurenmann RK. Factors influencing the efficacy of intraarticular steroid injections in patients with juvenile idiopathic arthritis. Eur J Pediatr 2008;167:425-30.

9. Huppertz HI, Tschammler A, Horwitz AE, Schwab KO. Intraarticular corticosteroids for chronic arthritis in children: efficacy and effects on cartilage and growth. J Pediatr 1995;127:317-21.

10. Zulian F, Martini G, Gobber D, Agosto C, Gigante C, Zacchello F. Comparison of intraarticular triamcinolone hexacetonide and triamcinolone acetonide in oligoarticular juvenile idiopathic arthritis. Rheumatology (Oxford) 2003;42:1254-9.

11. Zulian F, Martini G, Gobber D, Plebani M, Zacchello F, Manners P. Triamcinolone acetonide and hexacetonide intraarticular treatment of symmetrical joints in juvenile idiopathic arthritis: a doubleblind trial. Rheumatology (Oxford) 2004;43:1288-91.

12. Balogh Z, Ruzsonyi E. Triamcinolone hexacetonide versus betamethasone. A double-blind comparative study of the long-term effects of intraarticular steroids in patients with juvenile chronic arthritis. Scand J Rheumatol Suppl 1987;67:80-2.

13. Eberhard BA, Sison MC, Gottlieb BS, llowite NT. Comparison of the intraarticular effectiveness of triamcinolone hexacetonide and triamcinolone acetonide in treatment of juvenile rheumatoid 
arthritis. J Rheumatol 2004;31:2507-12.

14. Scherer J, Rainsford KD, Kean CA, Kean WF. Pharmacology of intraarticular triamcinolone. Inflammopharmacology 2014;22:201-17.

15. af Klint E, Grundtman C, Engström M, et al. Intraarticular glucocorticoid treatment reduces inflammation in synovial cell infiltrations more efficiently than in synovial blood vessels. Arthritis Rheum 2005;52:3880-9.

16. Petty RE, Southwood TR, Manners P, et al. International League of Associations for Rheumatology classification of juvenile idiopathic arthritis: second revision, Edmonton, 2001. J Rheumatol 2004;31:390-2.

17. Chakravarty K, Pharoah PD, Scott DG. A randomized controlled study of post-injection rest following intraarticular steroid therapy for knee synovitis. Br J Rheumatol 1994;33:464-8.

18. McCarty DJ, Harman JG, Grassanovich JL, Qian C. Treatment of rheumatoid joint inflammation with intrasynovial triamcinolone hexacetonide. J Rheumatol 1995;22:1631-5.

19. Wallen M, Gillies D. Intraarticular steroids and splints/rest for children with juvenile idiopathic arthritis and adults with rheumatoid arthritis. Cochrane Database Syst Rev 2006:Cd002824.

20. Honkanen VE, Rautonen JK, Pelkonen PM. Intraarticular glucocorticoids in early juvenile chronic arthritis. Acta Paediatr 1993;82:1072-4.

21. Breit W, Frosch M, Meyer U, Heinecke A, Ganser G. A subgroup-specific evaluation of the efficacy of intraarticular triamcinolone hexacetonide in juvenile chronic arthritis. J Rheumatol 2000;27:2696702.

22. Lepore L, Del Santo M, Malorgio C, et al. Treatment of juvenile idiopathic arthritis with intraarticular triamcinolone hexacetonide: evaluation of clinical effectiveness correlated with circulating ANA and T gamma/delta + and B CD5 + lymphocyte populations of synovial fluid. Clin Exp Rheumatol 2002;20:719-22.

23. Batu ED. Glucocorticoid treatment in juvenile idiopathic arthritis. Rheumatol Int 2019;39:13-27.

24. Wallace CA. On beyond methotrexate treatment of severe juvenile rheumatoid arthritis. Clin Exp Rheumatol 1999;17:499-504.

25. Gotte AC. Intraarticular corticosteroids in the treatment of juvenile idiopathic arthritis: Safety, efficacy, and features affecting outcome. A comprehensive review of the literature. Open Access Rheumatol 2009;1:37-49.

\section{Figures}




\section{Strata $+\mathrm{TA}+\cdot \mathrm{TH}$}

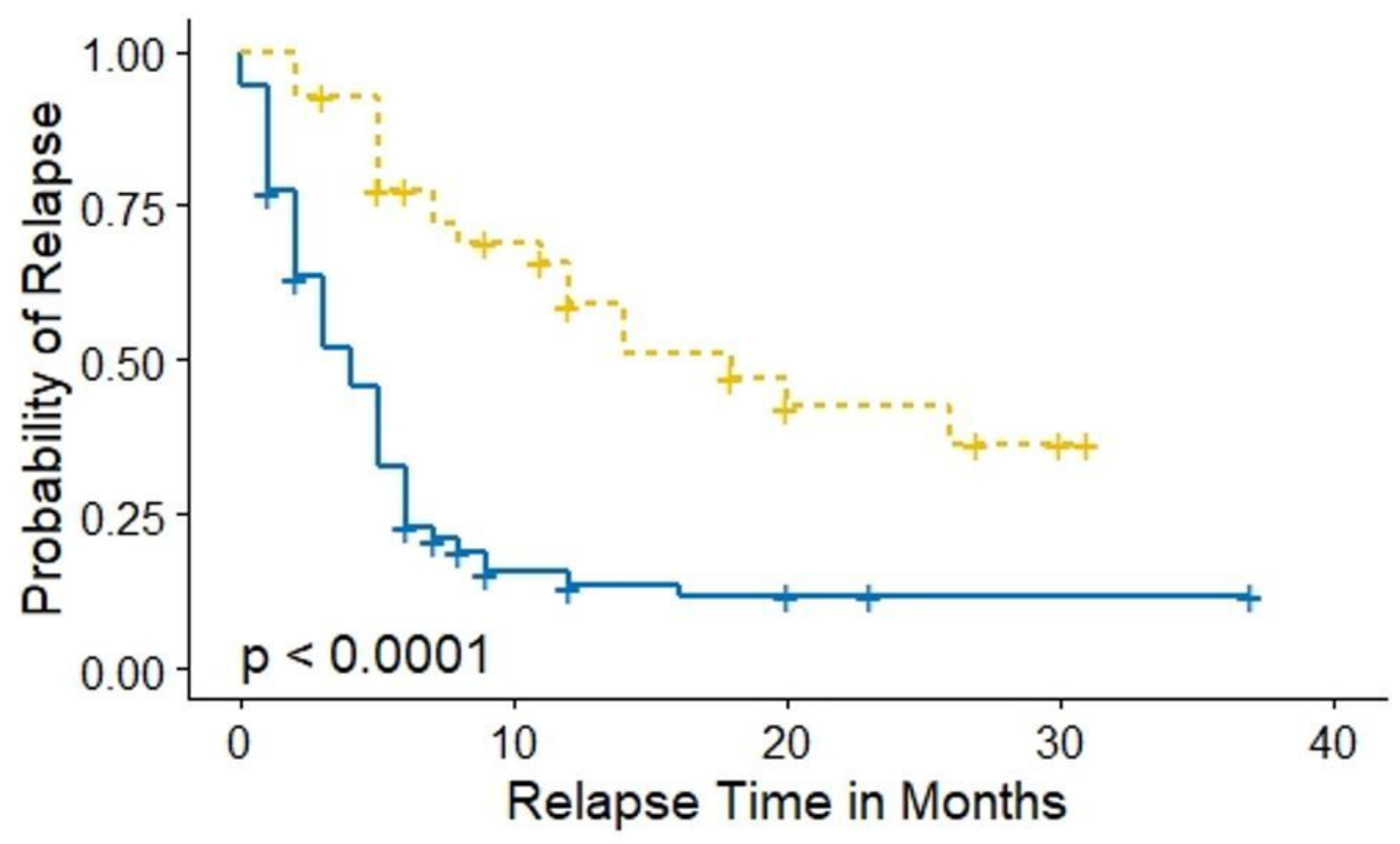

Figure 1

Kaplan Meier Curves by IAC Injection Type for All Joints. Censored values are denoted by a plus sign. The $p$-value in the figure indicates the p-value from log-rank test that compares the injections. 
Strata $+\mathrm{TA}+\cdot \mathrm{TH}$

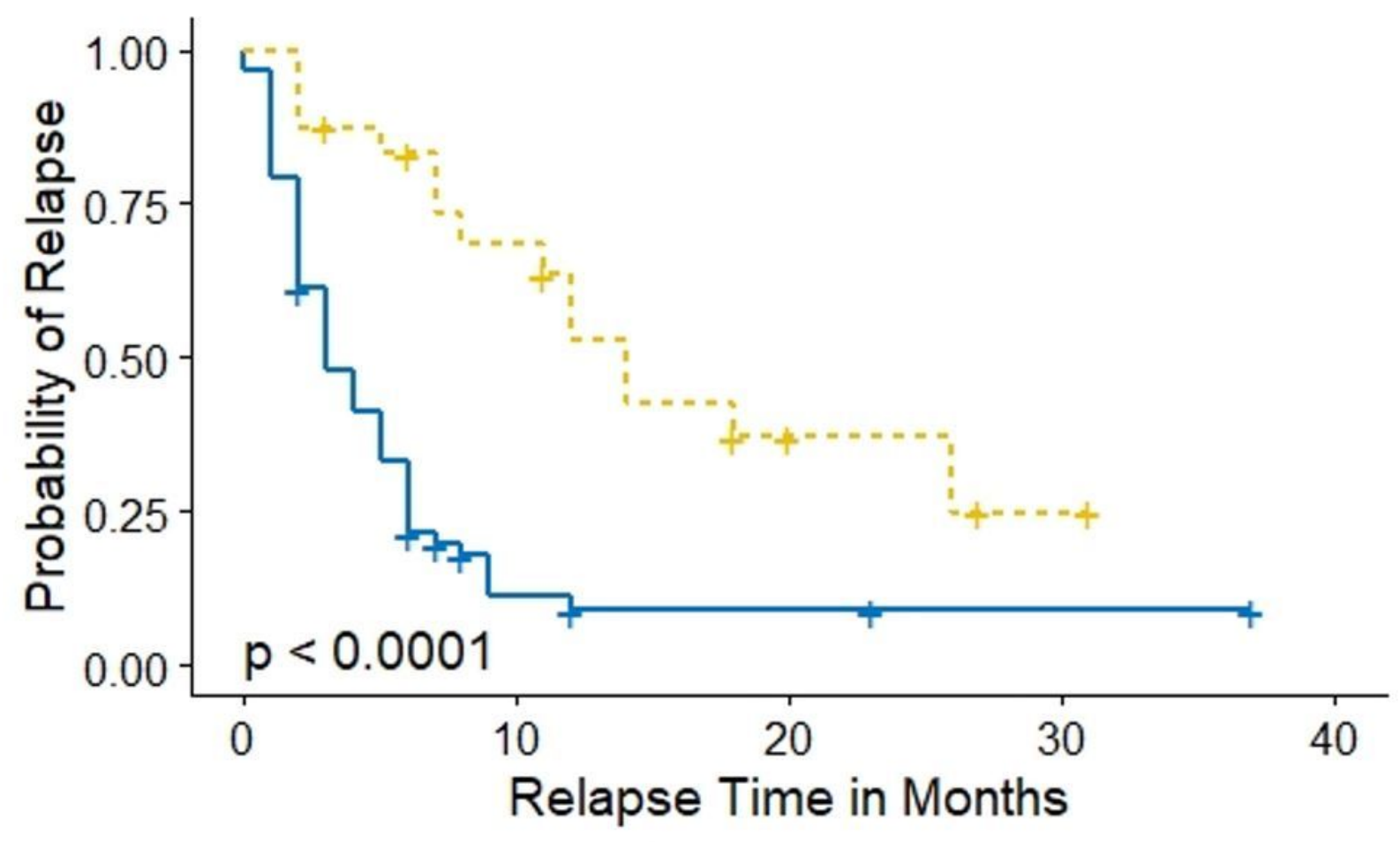

Figure 2

Kaplan Meier Curves by IAC Injection Type for Knee Only. Censored values are denoted by a plus sign. Pvalue listed in the figure represents the log-rank test $p$-value that compares the injection types. 